Ann. Biol. anim. Bioch. Biophys., 1977, 17 (5 B), 865-873.

\title{
Origine et transformation de l'acide palmitique des triglycérides du tissu adipeux brun du rat adapté au froid. Effet d'un régime hyperlipidique.
}

\author{
par Marie-Thérèse HLUSZKO, Claude SENAULT \\ Ecole Prafique des Hautes Ełudes, Laboratoire d'Adaptation Energétique à l'Environnement, \\ Collège de France, 11 Place Marcelin Berthelot, 75231 Paris Cedex 05.
}

Summary. Origin and transformation of triglyceride palmitic acid in the brown adipose tissue of the rat adapted to cold. Effect of a hyperlipidic diet.

Rats acclimated to $5^{\circ} \mathrm{C}$ received semi-synthetic diets containing either 5 p. 100 lipids (NL) or 26 p. $100(\mathrm{HL})$ with ${ }^{14} \mathrm{C}$-palmitic ocid or ${ }^{14} \mathrm{C}-\mathrm{U}$ glucose added as tracers. After one week of feeding labelled diets, brown adipose tissue (BAT), liver and neutral serum lipids reached isotopic balance. Desaturation and chain elongation of dietary palmitic acid and glucose fatty acid synthesis were studied in BAT triglycerides and compared to liver and serum.

When rats were fed the NL diet, the major fatty acids derived from dietary palmitic acid in the liver and in BAT were palmitoleic and stearic acids; they were found in equal amounts. Oleic acid was weakly labelled.

Palmitic acid was mostly synthesized from glucose ; oleic acid synthesis cccurred more readily de novo than from dietary palmitic acid.

It should be pointed out that de novo synthesis of stearic acid was greater in BAT than in the liver. Lipogenesis was considerably inhibited in liver and BAT in rats fed the HL diet. Dietary palmitic acid desaturation was abolished ; only a decreased elongation remained.

Fatty acid turnover studies suggest that palmitic acid in BAT could be largely oxidized for thermogenesis, whereas oleic acid, the main triglyceride component, could be a stored fatty acid.

\section{Introduction.}

Chez de nombreux mammifères, on sait que le tissu adipeux brun joue un rôle important dans la thermogenèse sans frisson. Cette thermogenèse est sous contrôle neuro-humoral dont le principal effecteur est la noradrénaline. En conséquence, en liaison avec l'action lipolytique de l'hormone, la thermogenèse du tissu adipeux brun est due essentiellement à une stimulation de son métabolisme lipidique (Drahota, 1970). Chez le rat adapté au froid, cette thermogenèse se substitue entièrement au frisson thermique (Bartunkova et al., 1971) ; le métabolisme lipidique du tissu adipeux brun se caractérise alors par un accroissement de la lipolyse et de l'oxydation 
mitochondriale des acides gras ainsi libérés (Prusiner et al., 1970), mais aussi par une lipogenèse in situ très active (Steiner et al., 1968).

Dans une précédente étude (Senault et Hluszko, 1975), nous avons montré que chez le rat adapté au froid, il est possible d'obtenir, en une semaine, un équilibre isotopique des lipides neutres du tissu brun interscapulaire, du foie et du sérum. Nous avons déterminé les proportions relatives des acides gras d'origine alimentaire et d'origine synthétique. Nous avons pu mettre en évidence, dans la graisse brune interscapulaire, en même temps qu'une captation des acides gras alimentaires, une synthèse in situ d'acides gras à partir du glucose. L'administration d'un régime hyperlipidique inhibe presque totalement les synthèses.

Dans ce travail, nous avons éfudié, chez le rat adapté au froid, en fonction de la teneur en lipides du régime, les transformations de l'acide palmitique du régime dans les acides gras des triglycérides du tissu adipeux brun ainsi que la synthèse des différents acides gras à partir du glucose.

\section{Matériel et méthodes.}

a) Animaux. Les expériences sont faites sur des rats mâles Long Evans adaptés à l'âge de 9 semaines dans une chambre climatique maintenue à $5^{\circ} \mathrm{C} \pm 1^{\circ} \mathrm{C}$.

b) Régimes. A l'âge de 10 semaines, les rats sont nourris ad libitum avec des régimes semi-synthétiques : un régime normolipidique (NL) contenant 5 p. 100 de lipides et un régime hyperlipidique $(\mathrm{HL})$ contenant 26 p. 100 de lipides. Leur composition détaillée a été précédemment publiée (Senault et al., 1975).

Pendant la $4^{\mathrm{e}}$ semaine d'adaptation thermique, les rats sont nourris avec les mêmes régimes additionnés soił d'acide palmitique ${ }^{14} \mathrm{C}-16 *$, soit de glucose ${ }^{14} \mathrm{C}-\mathrm{U}^{*}$. Les RAS des régimes marqués par l'acide palmitique ${ }^{14} \mathrm{C}-16$ sont respectivement de $1,80.10^{6} \mathrm{cpm} / \mathrm{g} A G$ du régime $N L$ et de $0,88.10^{6} \mathrm{cpm} / \mathrm{g}$ AG du régime $H L$. Le marquage du régime $N L$ par le glucose ${ }^{14} \mathrm{C}-U$ est de $58,8.10^{6} \mathrm{cpm} /$ carbone-g de glucose.

c) Techniques ef appareillage. Après sacrifice par décapitation, on recueille environ $6 \mathrm{mi}$ de sang, on prélève la graisse brune interscapulaire et un échantillon de foie de $3 \mathrm{~g}$ environ.

Les lipides totaux sont extraits par la méthode de Folch et al. (1957) et pesés.

Les lipides neutres : triglycérides et acides gras libres sont séparés sur couche mince. On utilise des plaques de verre de $20 \times 20 \mathrm{~cm}$ recouvertes de gel de silice Merck (PF 254) d'une épaisseur de 0,5 mm.

Les triglycérides et les acides gras libres sont récupérés par grattage et élués sur un verre fritté par $30 \mathrm{ml}$ de chloroforme-méthanol $1 / 1, \mathrm{v} / \mathrm{v}$. Après hydrolyse alcaline avec $\mathrm{KOH}$ alcoolique $0,5 \mathrm{~N}$ à $70^{\circ}$ pendant $1 \mathrm{~h}$, les acides gras libérés sont méthylés par du méthanol sulfurique.

Les acides gras sont étudiés par chromatographie gaz-liquide selon la technique de Bezard et al. (1964). Les colonnes ( 6 pieds, $1 / 4$ pouce) sont remplies par du chromosorb $W$ imprégné de DEGS à un taux de 25 p. 100. Un diviseur d'effluent à la

* Fournis par le C.E.A. 
sortie de la colonne permet un partage de 90/10,10 p. 100 servant à la détection des pics d'acides gras et à l'enregistrement d'un chromatogramme, 90 p. 100 servant au recueil des acides gras dans des capsules de verre. Les acides gras sont ensuite élués avec $10 \mathrm{ml}$ de mélange scintillant (PPO, POPOP, toluène). Les mesures de radioactivité sont faites sur un Tricarb Packard.

\section{Expression des résultats.}

Le pourcentage de la radioactivité porté par chacun des acides gras est calculé en faisant le rapport entre la radioactivité retrouvée sur chacun des pics et la radioactivité totale qui est égale à la somme de la radioactivité de chacun des pics. Connaissant la quantité d'acides gras injectée, la composition en acides gras du tissu et la radioactivité totale injectée, on détermine la radioacłivité spécifique (RAS) de chacun des acides gras.

\section{Résultałs.}

1) Lorsque l'on utilise l'acide palmitique comme marqueur (fig. 1), la majeure partie de la radioactivité se retrouve sur cet acide dans les trois tissus et pour les deux régimes.

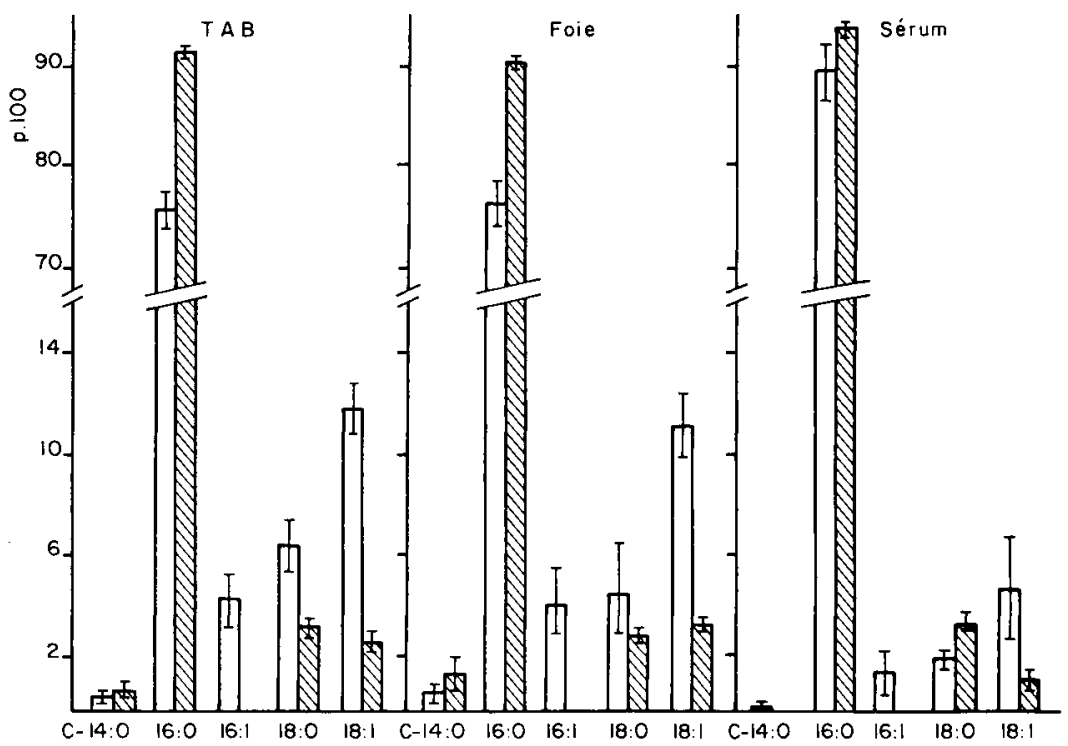

FIG. 1. - Répartition de la radioactivité totale dans les acides gras des triglycérides du tissu adipeux brun interscapulaire (TAB) du foie et du sérum. Les animaux maintenus à une température constante de $5^{\circ}$ durant 4 semaines reçoivent un régime à 5 p. 100 de lipides (NL) (blanc) ou 26 p. 100 de lipides (HL) (gris). Ces régimes sont additionnés d'acide palmitique ${ }^{14} \mathrm{C}-16$ à dose traceuse les 7 jours qui précèdent les mesures. Les acides gras des TG que l'on trouve marqués sont les acides myristique $(C-14: 0)$, palmitique $(C-16: 0)$, stéarique $(C-18: 0)$ et oléique $(C-18: 1)$ pour les 2 régimes. L'acide palmiloléique (C-16:1) n'est marqué que dans le cas du régime à $5 \mathrm{p}$. 100 de lipides. 4 rats par groupe (les valeurs moyennes sont accompagnées de l'écart standard). 
a) Dans le cas du régime NL, la radioactivité se répartit de la même façon dans le tissu adipeux brun et dans le foie : 75 p. 100 de l'activité totale se retrouve sur l'acide palmitique, le reste se répartissant sur les acides stéarique et palmitoléique en quantité voisine (5 à 6 p. 100) et, en plus forte proportion, sur l'acide oléique (12 p. 100). Le pourcentage de radioactivité retrouvé sur l'acide myristique est très faible.

Dans les deux tissus, c'est l'acide palmitique qui a la radioactivité spécifique la plus élevée (fig. 2) ; elle y est du même ordre de grandeur, leur composition en acides gras étant voisine. Les acides palmitoléique et stéarique ont des RAS voisines égales au $1 / 3$ de celle du marqueur dans la graisse brune et à la $1 / 2$ dans le foie. La radioactivité spécifique de l'acide oléique est beaucoup plus faible que celles des deux acides précédents ; elle est, dans le tissu adipeux brun, égale au 1/10 de celle de l'acide palmitique ef au 1/7 dans le foie. Dans les deux tissus, elle est voisine de celle de l'acide myristique.

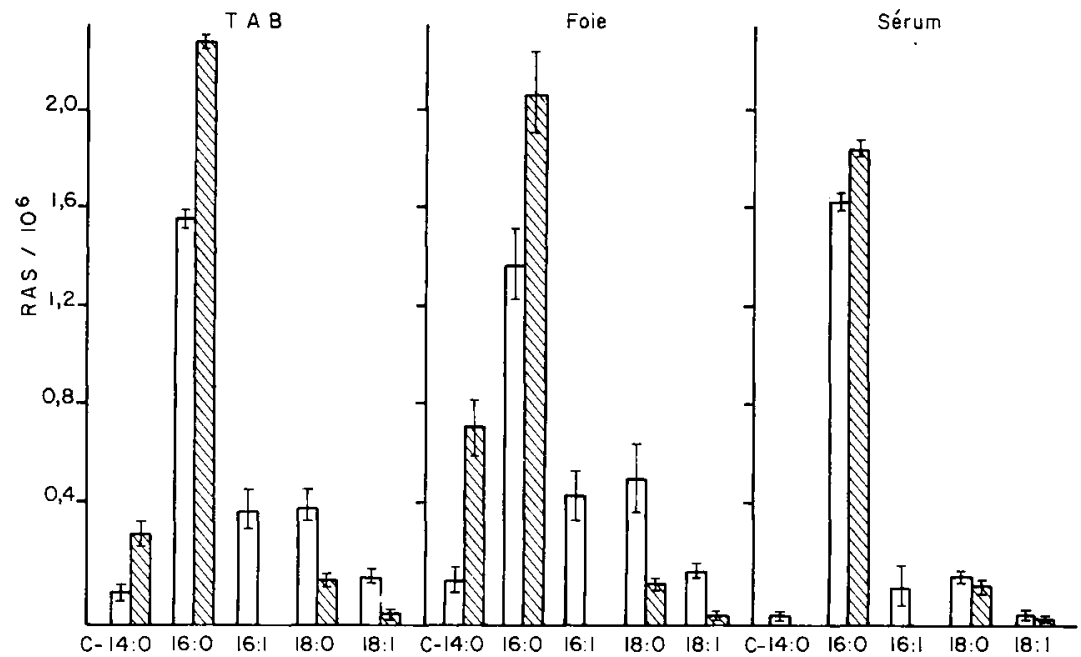

FIG. 2. - Radioactivité spécifique des acides gras des triglycérides du tissu adipeux brun interscapulaire (TAB) du foie et du sérum. Les animaux maintenus durant 4 semaines à un froid constant de $5^{\circ}$ reçoivent un régime à 5 p. 100 de lipides (NL) (blanc) ou 26 p. 100 de lipides (HL) (gris). Ces régimes sont additionnés d'acide palmitique ${ }^{14} \mathrm{C}-16$ pendant les 7 jours qui précèdent les mesures. Les RAS des régimes sont de $1,8010^{6} \mathrm{cpm} / \mathrm{g}$ d'AG (NL) et de $0,8810^{6} \mathrm{cpm} / \mathrm{g} \mathrm{d}$ 'AG (HL) ; celle des tissus de $0,5710^{6}$ (TAB), $0,5810^{6}$ (Foie) et $0,5810^{6}$ (Sérum) cpm/g d'AG. 4 rats par groupe (les valeurs moyennes sont accompagnées de l'écart standard).

Dans le sérum, le pourcentage de l'activité retrouvé sur l'acide palmitique (91 p. 100 de l'activité des acides gras du tissu) est significativement plus élevé que dans le foie et le tissu adipeux brun. Il en est de même pour la RAS de cet acide, celles des autres acides étant très faibles et même négligeables compte tenu de la dispersion des valeurs pour les acides myristique et oléique.

b) Dans le cas du régime $H L$, l'activité de l'acide palmitique représente 92 à 95 p. 100 de la radioactivité des acides gras totaux des trois tissus. Le reste se répartit sur les acides stéarique, oléique et myristique. Dans aucun cas, on ne retrouve de la radioactivité sur l'acide palmitoléique. 
Les RAS sont dans un ordre décroissant, la plus élevée pour l'acide palmitique, puis les acides myristique, stéarique et oléique.

2) Dans le cas de synthèse par condensation (régime NL marqué par du glucose ${ }^{14} \mathrm{C}-\mathrm{U}$ (fig. 3), l'acide palmitique demeure l'acide gras portant le pourcentage le plus élevé de la radioactivité totale (51 à 55 p. 100). Ce pourcentage est plus faible que dans le cas précédent; on ne note plus de différence entre les deux tissus et le sérum.

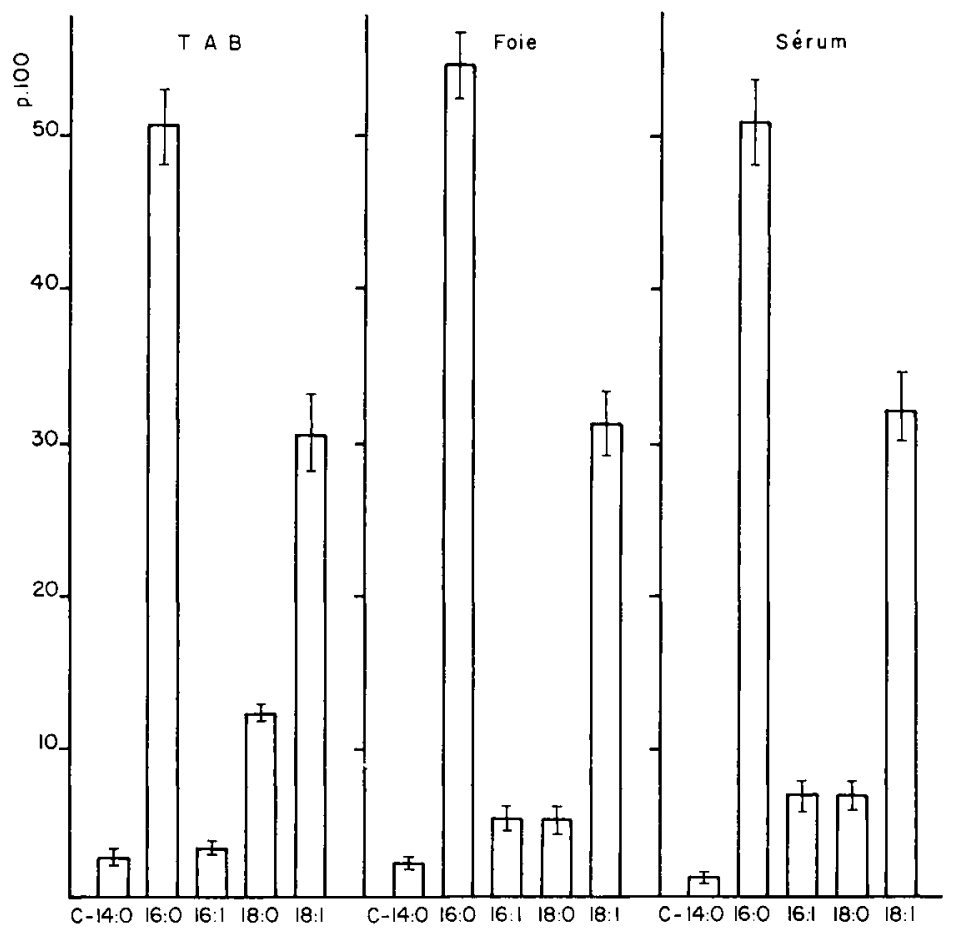

FIG. 3. - Répartition de la radioactivité tolale dans les acides gras synthétisés de novo: acides myristique ( $C-14: 0)$, palmitique $(C-16: 0)$, palmitoléique $(C-16: 1)$, stéarique $(C-18: 0)$ et oléique (C-18:1), des triglycérides du tissu adipeux brun interscapulaire (TAB), du foie, et du sérum. Les animaux maintenus durant 4 semaines à un froid constant de $5^{\circ}$, reçoivent pendant une semaine le régime à 5 p. 100 de lipides additionné, à dose traceuse, de ${ }^{14} \mathrm{C}-U$ glucose. 8 rats par groupe (les valeurs moyennes sont accompagnées de l'écart standard).

La répartition de la radioactivité sur les autres acides gras diffère avec le tissu. Les variations portent sur les acides stéarique et palmitoléique. Les pourcentages de radioactivité retrouvés sur ces deux acides sont égaux dans le foie et le sérum (5 ef 7 p. 100 en moyenne) alors que dans le tissu adipeux brun, il esł plus élevé pour l'acide stéarique (12 p. 100) que pour l'acide palmitoléique (3,5 p. 100). Quant aux acides myristique et oléique, les valeurs des pourcentages de radioactivité retrouvés sur ces deux acides varient peu avec le tissu. Ils sont, par contre, très augmentés par rapport au cas précédent où nous suivions essentiellement les transformations de l'acide palmitique du régime. 
Les radioactivités spécifiques des acides palmitique et oléique sont en équilibre (fig. 4), aussi bien dans le foie que dans le tissu brun interscapulaire. Dans ces deux tissus, les autres acides gras ont des radioactivités spécifiques semblables, excepté l'acide stéarique dont la RAS est significativement plus élevée dans le tissu adipeux brun que dans le foie. Elle est plus élevée que dans le cas précédent dans les trois tissus. De même, les RAS des acides gras autres que l'acide palmitique sont, dans leur ensemble, plus élevées dans le sérum.

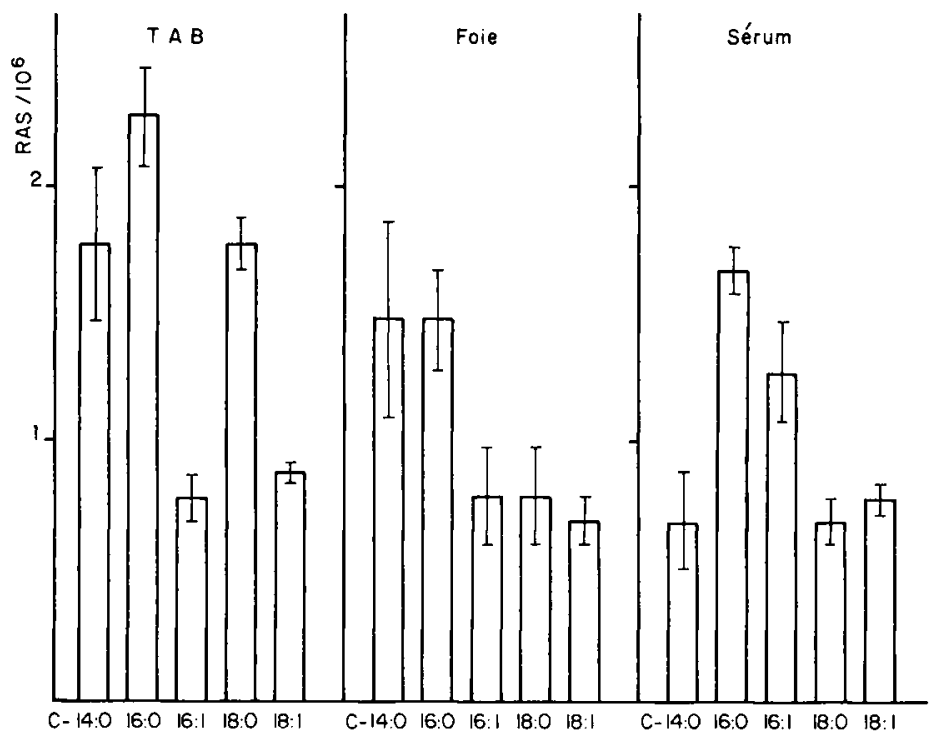

FIG. 4. - Radioactivité spécifique des acides gras des triglycérides du TAB, du foie ef du sérum, synthétisés de novo (glucose ${ }^{14} \mathrm{C}-U$ marqueur). 8 rats par groupe (les valeurs moyennes sont accompagnées de l'écart standard).

3) La décroissance de la radioactivité des acides gras marqués du tissu adipeux brun (tabl. 1), au cours des $24 \mathrm{~h}$ qui suivent la suppression de l'administration du marqueur acide palmitique (régime NL), montre que l'acide palmitique a le renouvellement

TABLEAU 1

Décroissance de la radioactivité des acides gras des triglycérides du TAB interscapulaire

C-16:0 C-16:1 C-18:0 C-18:1

Diminution de la radioactivité

(p. 100) en $24 \mathrm{~h}$

$+1 / 2(h)$

La décroissance de la radioactivité esł exprimée en p. 100 de la radioactivité des acides gras des TG au plateau d'équilibre. Ces valeurs sont celles obtenues $24 \mathrm{~h}$ après la suppression du précurseur radioactif ( 5 animaux). Les périodes de demi-vie sont délerminées graphiquement. 
le plus rapide $\uparrow 1 / 2<1$ jour $(16 \mathrm{~h})$ et que l'acide oléique a le renouvellement le plus lent $>4$ jours $(108 \mathrm{~h})$. Les acides palmitoléique et stéarique ont des vitesses de renouvellement intermédiaires avec des demi-vies voisines de $40 \mathrm{~h}$.

\section{Discussion.}

Cette étude est faite sur les rats adaptés au froid chez lesquels une semaine de maintien au régime marqué est suffisante pour obtenir un équilibre de l'ensemble des acides gras des triglycérides du tissu adipeux brun, du foie et du sérum ( 80 p. 100 des lipides de ces 3 tissus ont des demi-vies comprises entre 1,5 et 2 jours) (Hluszko et Senault, 1975). De ce fait, les résultats obtenus pour le tissu adipeux brun seront comparés à ceux obtenus pour le foie et le sérum. Dans ces conditions, les différences observées entre les tissus devront être considérées comme des caractéristiques du tissu ; l'effet de l'adaptation au froid ne pourra pas être évalué.

a) Devenir métabolique de l'acide palmitique.

L'activité des acides gras tissulaires, autres que l'acide palmitique, est due aux réactions d'élongation et de désaturation de cet acide et, également, aux synthèses par condensation des acetyls provenant du marqueur. Cependant, le métabolisme oxydatif étant très intense chez l'adapté au froid, nous sommes en droit de penser que la plus grande partie des acétyls produits au cours de la $\beta$ oxydation est oxydée dans le cycle de Krebs. L'activité de ces produits de condensation est alors négligeable, par rapport à celle des produits de transformation de l'acide palmitique.

On constate que la RAS de l'acide palmitique est, significativement, plus élevée dans le sérum que dans le foie et la graisse brune interscapulaire et que, dans ces 2 tissus, les RAS des acides palmitoléique et stéarique sont augmentées par rapport à celles existant dans le sérum. L'acide palmitique circulant capté par le tissu adipeux brun et par le foie est donc transformé in situ, soit par désaturation en acide palmitoléique C-16:1, soił par élongation en acide stéarique C-18:0. La comparaison des RAS de ces acides gras montre que ces transformations se font en proportions équivalentes et qu'elles sont quantitativement plus importantes dans le foie. Ces produits de transformation ne semblent être libérés dans la circulation qu'en très faibles quantités ; ils pourraient être utilisés in situ à des fins thermogénéfiques. Quant à l'acide oléique, il apparaît comme dérivant très peu de l'acide palmitique du régime. Les RAS de cet acide sont très faibles dans les 3 tissus. Le fait qu'elles soient inférieures à celle de l'acide stéarique laisse supposer que les processus d'élongation de l'acide palmitique ne seraient pas suivis de désaturation en quantité appréciable. Il est généralement admis que la désaturation des acides gras ne se fait que dans la fraction microsomale de la cellule alors que les enzymes d'élongation sont à la fois microsomales et mitochondriales. II est donc permis de penser que la plus grande radioactivité spécifique de l'acide stéarique par rapport à celle de l'acide oléique est dûe à une prépondérance de l'activité des systèmes d'élongation mitochondriaux. Avec le régime utilisé, l'acide oléique dériverait donc plus des synthèses par condensation que des transformations de l'acide palmitique.

L'effet inhibiteur sur la lipogenèse d'un régime hyperlipidique a été mis en 
évidence, in vivo (Jansen et al., 1966), et, in vitro, sur le foie et le tissu adipeux blanc (Lavau ef al., 1972 ; Zaragoza-Hermans, 1974). Dans une expérience précédente (Senault et Hluszko, 1975), nous avons retrouvé cet effet avec le régime HL sur le tissu adipeux brun. Les acides gras tissulaires sont, alors, essentiellement d'origine alimentaire. Cette étude nous montre que l'acide palmitique capté, se transforme très peu dans le tissu adipeux brun et dans le foie. Nous voyons d'autre part que ce sont les processus de désaturation qui sont les plus inhibés. II n'y a plus de désaturation de l'acide palmitique et il se forme 4 fois moins d'acide oléique que dans le cas du régime NL. Il y aurait essentiellement une inhibition des systèmes enzymatiques microsomaux de désaturation, mais aussi, à moindre degré, de ceux d'élongation. Cette plus grande sensibilité de l'effet inhibiteur du régime HL sur les enzymes de la fraction microsomale laisse supposer que les deux voies métaboliques de l'élongation pourraient avoir un rôle physiologique différent, comme l'avaient déjà suggéré Seubert et Podack (1973). Pour ces auteurs, la fonction de la voie d'élongation microsomale serait de fournir des acides gras à chaîne plus longue, alors que celle de la voie mitochondriale serait surtout de maintenir disponible en permanence des NADP pour la néoglucogenèse. Notre régime étant riche en protéines, cette voie garderait une certaine activité.

\section{b) Synthèse à partir du glucose}

Dans le cas des synthèses par condensation, on observe que l'acide oléique $a$, dans les 3 tissus étudiés, une RAS deux fois plus faible que celle de l'acide palmitique (alors qu'elle était 10 à 20 fois plus faible avec l'acide palmitique comme marqueur). Ceci tend à confirmer que la partie de l'acide oléique, d'origine synthétique, résulte moins des processus de transformation de l'acide palmitique que de synthèse par condensation. Cependant, c'est la RAS de l'acide palmitique qui demeure la plus élevée dans les 3 tissus ; c'est donc l'acide gras dont la synthèse de novo est la plus importante.

Quant aux autres acides gras, nous avons vu que, dans le foie, ils ont des RAS égales. Les pourcentages de radioactivité retrouvés sur les acides palmitoléique et stéarique sont semblables à ceux trouvés lorsque c'est l'acide palmitique qui est marqué. Dans le foie, ces deux acides gras dériveraient donc essentiellement de l'acide palmitique, qu'il soit de synthèse ou alimentaire. II en serait de même pour l'acide palmitoléique dans le tissu adipeux brun interscapulaire. Par contre, dans ce tissu, on retrouve, sur l'acide stéarique, un pourcentage de la radioactivité totale plus important que précédemment. La RAS de cet acide est, de ce fait, plus élevée et peu différente de celle des acides myristique et palmitique. Une partie des acides gras tolaux du tissu adipeux brun étant synthétisée in sifu (Senault et Hluszko, 1975), la synthèse de novo porterait plus spécifiquement sur l'acide stéarique. Cet acide aurait, dans le tissu, une double origine synthétique : élongation de l'acide palmitique qui serait plus mitochondriale que microsomale, et synthèse par condensation.

L'étude de la décroissance au cours des $24 \mathrm{~h}$ qui suivent la suppression du marqueur nous montre que l'acide palmitique est l'acide gras des triglycérides dont le renouvellement est le plus rapide dans le tissu brun interscapulaire. II semble donc être l'acide gras préférentiellement oxydé pour les besoins énergétiques de ce tissu 
qui, chez l'adapté au froid, se caractérise par un métabolisme lipidique intense. L'utilisation préférentielle de l'acide palmitique pour la thermogenèse peut être une des causes de l'allongement de la chaîne carbonée moyenne des triglycérides du tissu adipeux brun chez les adaptés au froid ainsi que chez les animaux nourris avec le régime HL (Senault et al., 1975). L'estérification des triglycérides par l'acide oléique se fait alors dans des proportions plus importantes. L'acide oléique qui a un renouvellement plus lent pourrait avoir surtout un rôle d'acide gras de réserve.

Réunion Groupe Développement INRA/Productions animales Thiverval-Grignon, 14-15 avril 1976.

Remerciements. - Nous remercions J. Lecerf qui, dans le Laboratoire du Professeur Bezard, nous a initiés à la technique de récupération des esters élués par chromatographie en phase gazeuse.

\section{Références}

BARTUNKOVA R., JANSKY L., MEJSNAR J., 1971. Nonshivering thermogenesis and cold adaptation. PP. 39-56, In JANSKY L. Nonshivering Thermogenesis, Academia Prague.

BEZARD J., BOUCROT P., CLEMENT G., 1964. Collecte d'esters d'acides gras marqués au tritium ef au carbone-14 élués par chromatographie gaz-liquide. J. Chromatog., 14, 368-377.

DRAHOTA Z., 1970. Fatty acid oxydation by brown adipose fissue mitochondria, 225-244. In LIND. BERG O., Brown adipose tissue, Elsevier, N. Y.

FOLCH J., LEE S. M., SLOANE-STANLEY G. M., 1957. A simple method for the isolation and purification of total lipids from animal tissues. J. biol. Chem., 226, 497-509.

HLUSZKO M. T., SENAULT C., 1975. Effet de l'adaptation au froid sur la vitesse de renouvellement des acides gras des triglycérides du tissu adipeux brun. J. Physiol. Paris, 71, $289 \mathrm{~A}$.

JANSEN G. R., HUTCHISON C. F., ZANETTI M. E., 1966. Lipogenesis in vivo. Effect of dietary fat or starvation on conversion of ${ }^{14} \mathrm{C}$-glucose into fat and turnover of newly synthetized fat. Biochem. J., 99, 323-340.

LAVAU M., NADEAU M., SUSINI C., 1972. Métabolisme in vitro du tissu adipeux épididymaire du rat en éłat d'obésité nutritionnelle. Biochimie, 54, 1057-1067.

PRUSINER S., CANNON B., LINDBERG O., 1970. Mechanisms controlling oxidative metabolism in brown adipose tissue, 283-318. In LINDBERG O., Brown adipose fissue, Elsevier, N. Y.

SENAULT C., HLUSZKO M. T., PORTET R., 1975. Effect of diet and cold acclimation on lipid composition of rat interscapular brown adipose tissue. Ann. Nutr. Alim., 29, 67-77.

SENAULT C., HLUSZKO M. T., 1975. Etude de l'origine lipidique ou glucidique des acides gras des triglycérides du tissu adipeux brun du rat adapté au froid. J. Physiol. Paris, 71, 312 A.

SEUBERT W., PODACK E. R., 1973. Mechanisms and physiological roles of fatty acid chain elongation in microsomes and mitochondria. Mol. Cell. Biochem., 1, 29-40.

STEINER G., SCHÖNBAUM E., JOHNSON G. E., SELLERS E. A., 1968. Lipid metabolism : effects of immunosympathectomy and acclimation to cold. Can. J. Physiol. Pharmacol., 46, 453-461.

ZARAGOZA-HERMANS, N. M., 1974. Studies on the metabolic effects induced in the rat by a high fat diet. Control of glucose metabolism in adipose tissue of fed and fasted rats. Eur. J. Bioch., 48, 579-582. 